\title{
Standardization in optics characterization
}

Ristau, Detlev, Balasa, Istvan, Jensen, Lars

Detlev Ristau, Istvan Balasa, Lars Jensen, "Standardization in optics characterization," Proc. SPIE 10805, Laser-Induced Damage in Optical Materials 2018: 50th Anniversary Conference, 108050A (16 November 2018); doi: $10.1117 / 12.2502253$

SPIE. Event: SPIE Laser Damage, 2018, Boulder, Colorado, United States 


\title{
Standardization in Optics Characterization
}

\author{
Detlev Ristau $^{1,2^{*}}$, Istvan Balasa ${ }^{1}$, Lars Jensen ${ }^{1}$, \\ 1: Laser Zentrum Hannover e.V., Hollerithallee 8, 30419 Hannover, Germany \\ 2: Leibniz Universität Hannover, Welfengarten 1, 30167 Hannover, Germany
}

\begin{abstract}
An adapted quality management is a major prerequisite for the reliable production of optical components and coatings. Especially laser applications impose highest demands on modern optical systems and can be considered as a major pacemaker for the development of advanced quality management strategies. Therefore, most of the standardized concepts for the determination of the properties necessary for a comprehensive quality control, as for example losses and transfer functions as well as laser-induced damage thresholds or defect densities of optical surfaces, are based on laser systems. This contribution is intended to offer a brief review on the present status of optics characterization and the related standards often applied in the production of laser components. A selection of International Standards will be presented with focus on the determination of laser induced damage thresholds (ISO 21254), optical absorption (ISO 11551), and Total Scattering (ISO 13696). The corresponding measurement methods will be described and discussed before the background of recent developments in laser technology. Finally, some aspects of the future development and projects of international standardization activities will be discussed.
\end{abstract}

Keywords: standardization, laser damage ISO 21254, total scattering ISO 13696, absorption ISO 11551, ISO 13142, ISO 15368, ISO 13697

\section{INTRODUCTION}

In many fields of modern laser technology progress is directly dependent on reliable and standardized characterization procedures for the applied system components. Especially, optical components play a key role in the design of complex laser systems and often limit the potential of new products and applications. Prominent examples can be found in laser material processing, medical applications, or micro-lithography, where the economic efficiency is closely coupled to the optical losses and the lifetime of optical systems. In many fields of research and development, optical components have to fulfil a variety of critical requirements, which can be only achieved on the basis of advanced production and characterization techniques. And further, for many pioneering experiments in fundamental physics on laser fusion, gravitational wave detection, or the generation and application of ultrashort pulses, high quality optical components with outstanding power handling capabilities and extremely low losses are essential for the success.

In this context, standardized optics characterization techniques are of crucial importance: They are the basis for a clear specification and comparison of the quality parameters, which are necessary for the design and implementation of every optical system. Furthermore, for the optimization, production, and commercialization of optical components within the global market, standardized measurement concepts have to be available. Therefore, in most optics companies and research institutes, according characterization techniques are considered as strategic tools for quality management within the production line and research activities. In the course of the rapid development of optics characterization techniques during the last three decades, working groups within the International Organization for Standardization (ISO) have been initiated, which are dedicated to the standardization of measurement procedures for the quality assessment of optical laser components.

In the present outline, selected standards for optics characterization of widespread use will be summarized and discussed. The focus will be placed on the optical losses and the laser induced thresholds of laser components as major properties governing their versatility in the application. The underlying standardized measurement techniques will be briefly described, and aspects for revision of the standards in respect to recent developments in laser technology will be reviewed. Finally, recent trends in the standardization of optics characterization will be considered.

*d.ristau@1zh.de; phone+49 511 2788-240; fax +49 511 2788-100; lzh.de

Laser-Induced Damage in Optical Materials 2018: 50th Anniversary Conference, edited by Christopher Wren Carr, Gregory J. Exarhos, Vitaly E. Gruzdev, Detlev Ristau, M.J. Soileau, Proc. of SPIE Vol. 10805, 108050A (c) 2018 SPIE · CCC code: 0277-786X/18/\$18 - doi: 10.1117/12.2502253 


\section{QUALITY PARAMETERS FOR OPTICAL LASER COMPONENTS}

Selected quality parameters and corresponding measurement standards frequently considered in optical technology are compiled in Table 1. Besides the determination of the spectral behavior of the reflectance and transmittance according to ISO 15368 [1], a standard ISO 24013 [2], for the determination of the phase shift between the polarization components induced by phase retardation mirrors had been developed. Several measurement standards are dedicated to the measurement of optical losses including absorptance, scattering and total losses. The application of laser calorimetry for a calibrated determination of optical absorption is well established and described in ISO 11551 [3]. Especially for scatter losses, a variety of national and international standards were developed during the last three decades. As the most prominent ISO Standard for a characterization of optical components in respect to their Total Scattering (TS), ISO 13696 can be considered [4], which is based on an integration or collection of radiation scattered by the sample. Since this standard is concentrated on relatively sensitive techniques involving predominantly laser sources, a new standardization project, ISO/CD 19962, based on spectrophotometric scatter measurements is presently under discussion and reached the state of an ISO Committee Draft. In view of various research activities focused on the detailed scatter behavior of optical components, also a new work item for angle resolved scattering, ISO/WD 19986, had been initiated. As a consequence of the increasing demand for optical coatings with extremely low total losses, a cavity ring down standard, ISO 13142 [5], had been established in the year 2015.

Table 1: Selected quality parameters of optical laser components and the corresponding characterization techniques.

\begin{tabular}{|c|c|c|c|c|}
\hline Specification & \multicolumn{2}{|l|}{ Parameter / Unit } & \multicolumn{2}{|c|}{ Standard / Measurement Principle } \\
\hline $\begin{array}{l}\text { Transfer } \\
\text { Function }\end{array}$ & $\begin{array}{l}\text { Reflectance } \\
\text { Transmittance } \\
\text { Phase }\end{array}$ & $\begin{array}{l}\% \\
\% \\
\circ\end{array}$ & $\begin{array}{l}\text { ISO 15368: } \\
\text { ISO 13697: } \\
\text { ISO 24013: }\end{array}$ & $\begin{array}{l}\text { spectrophotometry } \\
\text { precise ratiometric method } \\
\text { phase retardation }\end{array}$ \\
\hline Optical Losses & $\begin{array}{l}\text { Absorptance } \\
\text { Scattering } \\
\text { Scattering } \\
\text { TS } \\
\text { Total Losses }\end{array}$ & $\begin{array}{l}\text { ppm } \\
\% \\
\text { ppm } \\
\text { ppm } \\
\text { ppm }\end{array}$ & $\begin{array}{l}\text { ISO 11551: } \\
\text { ISO/CD 19962: } \\
\text { ISO/WD 19986 } \\
\text { ISO 13696: } \\
\text { ISO 13142: }\end{array}$ & $\begin{array}{l}\text { laser calorimetry } \\
\text { spectroscopic measurement } \\
\text { angle resolved scattering } \\
\text { total scattered radiation } \\
\text { cavity ring down }\end{array}$ \\
\hline $\begin{array}{l}\text { Laser Induced } \\
\text { Damage } \\
\text { Threshold }\end{array}$ & $\begin{array}{l}\text { cw-LIDT } \\
1 \text { on 1-LIDT } \\
\text { S on 1-LIDT } \\
\text { Certification }\end{array}$ & $\begin{array}{l}\mathrm{W} / \mathrm{cm}^{2} \\
\mathrm{~J} / \mathrm{cm}^{2} \\
\mathrm{~J} / \mathrm{cm}^{2} \\
\mathrm{~J} / \mathrm{cm}^{2}\end{array}$ & $\begin{array}{l}\text { ISO 21254-1: } \\
\text { ISO 21254-2: } \\
\text { ISO 21254-3: } \\
\text { ISO/TR 21254: }\end{array}$ & $\begin{array}{l}\text { definition and principles } \\
\text { damage threshold measurement } \\
\text { certification power handling } \\
\text { damage detection }\end{array}$ \\
\hline $\begin{array}{l}\text { Surface } \\
\text { Quality }\end{array}$ & $\begin{array}{l}\text { Form-Tolerance } \\
\text { Scratches, Digs, } \\
\text { Roughness } \\
\text { Coating Defects } \\
\text { Contamination }\end{array}$ & $\begin{array}{l}\lambda / \mathrm{N} \\
5 / \mathrm{NxA} \\
\mathrm{nm}_{\mathrm{rms}}\end{array}$ & $\begin{array}{l}\text { ISO 10110: } \\
\text { ISO 10110: } \\
\text { ISO 9211-1: } \\
\text { ISO/TR 20811 }\end{array}$ & $\begin{array}{l}\text { part } 5 \text {, interferometry } \\
17(15 \text { active) parts containing } \\
\text { different types of imperfections } \\
\text { different types of defects } \\
\text { qualification for space applications }\end{array}$ \\
\hline Stability & $\begin{array}{l}\text { Abrasion } \\
\text { Environmental } \\
\text { Stability }\end{array}$ & & $\begin{array}{l}\text { ISO 9211: } \\
\text { ISO 9022: }\end{array}$ & $\begin{array}{l}\text { different test methods } \\
>\quad 20(15 \text { active }) \text { parts containing } \\
\text { a variety of conditioning methods }\end{array}$ \\
\hline
\end{tabular}


The standardization of Laser Induced Damage Thresholds (LIDT) had been a vibrant topic over more than forty years and resulted in a series of standards dedicated to different operation conditions for LIDT measurements. The first series, ISO 11254 [6], had been published at the beginning of the century, however it underwent a significant restructuring during the following years and is presently available in its revised form, ISO 21254 [7], which is divided into four parts. Further non-optical quality parameters are defined in ISO 10110 [8] which was created for a standardized preparation of drawings for optical elements and systems. In many applications, also the stability of the components in respect to environmental, mechanical, and chemical influences has to be considered. For this purpose, the standards ISO 9022 [9] and ISO 9211 [10] describe a broad spectrum of different conditioning methods including climatic influences, contamination, and mechanical stress as well as chemical agents, abrasion, and adhesion tests. Finally, a technical report ISO/TR 20811 [11] on contamination effects of optical components for space applications, which had been published recently, shall be mentioned. In the following the underlying measurement principles of a few selected characterization standards will be outlined.

\section{OUTLINE OF SELECTED STANDARD MEASUREMENT METHODS}

\subsection{Laser Calorimetry according to ISO 11551}

In the context of most measurement principles, the absorptance of an optical laser component is defined by the fraction of the impinging laser power converted into heat, which dissipates in the bulk of the component and induces thermal distortion effects. Absorption in laser optics can be often attributed to absorbing defects, inclusions, or stoichiometric deficiencies of the coating and optical bulk material, as well as to contamination or adsorbed species at the surfaces or interfaces of the deposited layer systems. Besides the thermal distortion, which may affect the application, optical absorption always implies the loss of laser energy, which is produced with high economical and technical expense. The measurement and optimization of absorptance is therefore of primary concern for the production and application of high quality optical components since the beginning of optical components and especially, coating production.

During the first decades of research in high quality laser coatings, absorption measurement were primarily performed on the basis of photothermal deflection schemes [12] which offer several advantages as for example low complexity, fast response time, high spatial resolution, and sensitivity limits below $1 \mathrm{ppm}$. However, as results of intensive studies, specific difficulties of the photothermal deflection techniques were observed, which impose severe difficulties on standardized absorption measurements via photothermal deflection: The deflection signal is dependent on a broad spectrum of sample parameters including the thermo-elastic and structural properties, which have to be considered for the calibration of the method. Also, during testing of coated samples, various complicated and unpredictable shift effects were observed.

In comparison to deflection techniques, laser calorimetry [13] is well adapted to the task: The absorption can be directly derived from a few parameters including the temporal behavior of the measured temperature signal, the power of the pump laser beam and the heat capacity of the sample. Consequently, the current International Standard for the determination of absorptance, ISO 11551, has been based on laser calorimetry. In the course of a calorimetric measurement cycle, the specimen is mounted in a thermally isolated chamber and aligned in respect to the pump laser beam. When the sample has reached thermal equilibrium with the environment at the time instant $t_{1}$, it is irradiated with the pump laser beam, and the temperature rise, which is induced by the absorbed laser power, is monitored with a temperature sensor. After a certain irradiation time, the laser is blocked at the time $t_{2}$, and the temperature of the specimen is monitored over a cooling period. For determination of the absorptance value, the resulting temperature curve is evaluated according to the methods described in ISO 11551. In first order, assuming ideal thermal properties of the test component, the heating $\mathrm{T}_{\mathrm{H}}(\mathrm{t})$ and the cooling $\mathrm{T}_{\mathrm{C}}(\mathrm{t})$ curves can be modelled by exponential functions with a small number of parameters:

$$
T_{H}(t)=T\left(t_{1}\right)+\frac{\alpha P}{\gamma C_{e f f}}\left(1-e^{-\gamma\left(t-t_{1}\right)}\right) \quad T_{C}(t)=T\left(t_{1}\right)+\frac{\alpha P}{\gamma C_{\text {eff }}}\left(e^{-\gamma\left(t-t_{2}\right)}-e^{-\gamma\left(t-t_{1}\right)}\right)
$$

Where $\mathrm{P}$ is the power of the pump laser, $\gamma$ describes the heat loss factor of the sample to the environment, and $\alpha$ symbolizes the absorptance to be determined. $\mathrm{C}_{\text {eff }}$ denotes the effective heat capacity of the sample with the contribution of the mount, which can be neglected for optimized holder geometries. Employing one of the methods for the determination of the absorptance value recommended in ISO 11551, the exponential curves are fit to the measured temperature behavior attaining the coefficient $\gamma$ and the factor $\alpha \mathrm{P} / \gamma \mathrm{C}_{\text {eff. }}$. The power of the pump laser can be measured directly, and 
the heat capacity of the sample is determined by weighing and extracting the specific heat of the bulk material from tables. Thus, the absorptance of the sample can be derived on the basis of well-defined parameters. For calibration of the measurement, different schemes based on reference samples of known absorptance, electrical heating with a specified energy dose, or by a direct calibration of the temperature sensors can be applied.

The practicability of the standard was verified in international round robin tests. For example good agreement of the absorptance data within an extended round-robin experiment [14], which involved more than 400 single measurements and approximately 100 samples, clearly indicated the versatility of ISO 11551 for the wavelength of $10.6 \mu \mathrm{m}$ of the $\mathrm{CO}_{2}$ laser. Similar campaigns were also accomplished for the wavelength of the Nd:YAG-laser operating at $1.064 \mu \mathrm{m}$ [15] and revealed significant discrepancies of the measured absorptance values. In view of these observations, an extended investigation in the standard measurement principle was carried out. The low thermal conductivity values of glass and quartz materials, which are routinely employed for the near infrared and visible spectral region, were found to be in contradiction to the elementary assumptions for the evaluation schemes given in the standard. Consequently, the heat transport equation has to be solved under consideration of a finite thermal diffusivity of these components and all boundary conditions for the calorimetric measurement procedure. A major practical aspect is the temporal development of the temperature profile on the sample surface revealing a delay between the start of irradiation and the temperature increase at the location of the attached sensor. Vice versa, the measured temperature curve reaches its maximum with a certain delay after blocking the laser irradiation. These retardation effects result in a dependence of the temperature signal and the final measurement value on the position of the sensor. However, a systematic analysis indicates clear requirements concerning the optimum sensor position for specific diameters of the typically disc shaped specimens, and the effect of finite thermal conductivity can therefore be compensated in practice. Another difficulty of calorimetric absorptance measurements in the NIR/VIS-spectral range is the low absorption level of high quality components in the range between less than 1 up to $100 \mathrm{ppm}$, which imposes high demands on the sensitivity of the calorimeter. For typical output power values of the pump lasers around a few ten Watts, temperature differences in the mK-region have to be clearly resolved. During the last two decades, enormous progress has been achieved $[16,17]$ for the sensitivity of laser calorimetric facilities which could be improved to a level below $1 \mathrm{ppm}$ and reaches limit values of $0.02 \mathrm{ppm}$ today (see for example Fig. 1). As a consequence of these research results, modifications of the original standard in respect to an improvement of the accuracy and sensitivity were performed, and a revised version of ISO 11551 [3] was published in the year 2003.

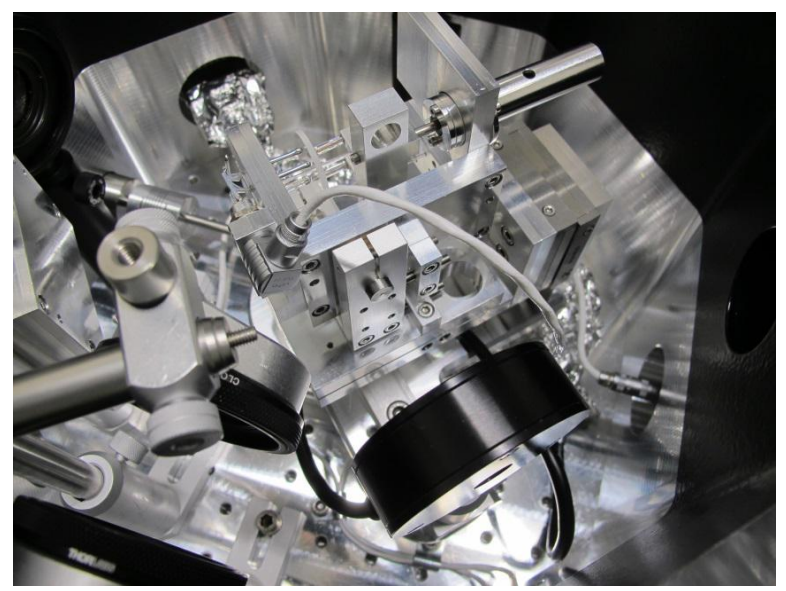

Fig. 1: View into the measurement compartment of a laser calorimeter according to ISO-11551 for the DUV/VUV spectral range. For minimum thermal contact to the holder, the sample is clamped directly in-between the temperature sensors and supported by small glass rods at the bottom section.

In the course of the development of optical coatings and components for the DUV-lithography, further laser calorimetric investigations indicated nonlinear absorption and preconditioning effects of the samples. Since the photon energy of the applied DUV/VUV lasers is relatively near to the bandgap of the employed optical materials, an increase of optical absorption with irradiation power directly related to two photon absorption effects [18 - 20] was observed. Also, various influences of the measurement environment and surface contaminations were detected [21,22] and analyzed for the different application areas of optical components down to the VUV spectral range. These experimental findings and some 
further adjustments to recent developments in laser technology are subject of a second revised version of the laser calorimetry standard which is presently in the state of a Draft International Standard (DIS) and will probably be published in the upcoming year 2019. In the meantime, additional aspects in respect to the geometry of the specimens moved into the focus of current research activities [23]. Modern laser systems contain a variety of components with rectangular, rod shaped or even more complex geometries, which have to be qualified in respect to their absorption behavior. Since these components may feature temperature profiles completely differing from the typical heat distribution in disc shaped samples, the present version of ISO 11551 is not applicable. Accordingly, a further revision of the standard in respect to additional sample geometries is arranged for the upcoming years.

a)
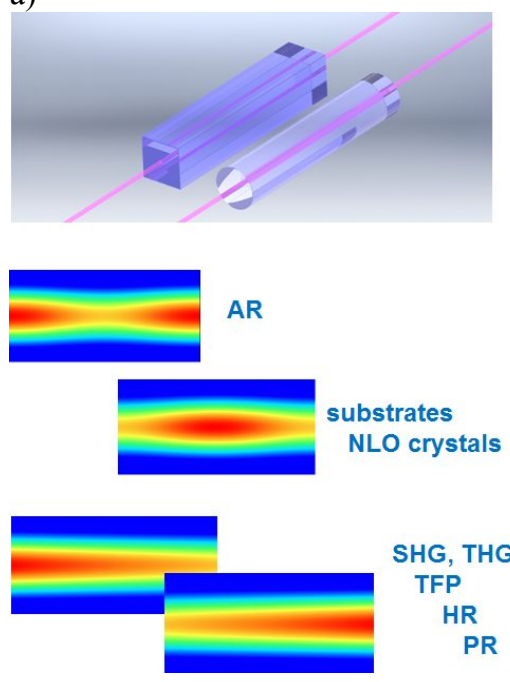

SHG THG

TFP

HR

PR b)

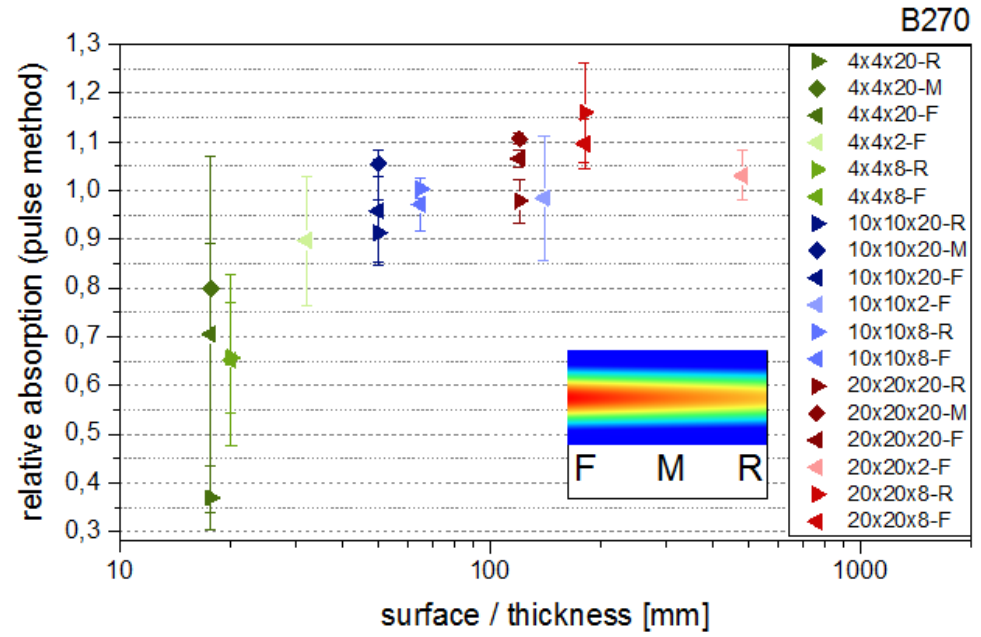

Fig. 2: Principal heating schemes of different optical components upon laser irradiation (a). Dependence of determined absorption value on the detector position for different sample geometries, utilizing B270 as substrate material. Summary of data from [60].

\subsection{Measurement of optical scattering}

Optical scattering is a loss mechanism of primary importance for optical laser components and their applications. Scattering reduces the efficiency of an optical system, and it deteriorates its imaging quality or the resolution. Especially in high power laser technology, scattering may also lead to severe safety problems, because even a low fraction of scattered radiation results in hazardous laser power levels in the environment endangering the operator. Scattering in optical components is mainly governed by the surface roughness of the functional surfaces as well as defects or inclusions in the coatings and the bulk materials. Especially, for the assessment of surface roughness by scatter measurements and stylus techniques, an enormous scientific and technical background has been built up in the 1980es [24, 25] and also motivated the development of National Standards for the measurement of total integrated (ASTM F1048 [26]) and angle resolved (ASTM E139236 [27]) scattering in the United States of America. After an extended discussion on a measurement parameter of practical importance and adequate scatter measurement schemes, ISO 13696 had been elaborated by ISO/TC 172/SC 9/WG 6 "Optics and Photonics - Electro-optical Systems - Optical components and their test methods" [4] and published in the year 2002. In this standard, total scattering (TS) is defined as the total amount of radiation scattered by an optical component into the $4 \pi$-fullspace and represents a quality parameter without relation to other characteristics of the specimen. The measurement concepts described in ISO 13696 are based on a collection or integration of the scattered radiation by an Ulbricht- [28] or a Coblentz- [29] sphere and a recording of the total amount of scattered radiation power. In order to gain a statistical basis, a line scan or mapping of the test surface is performed, and the measurement data are evaluated in respect to the elementary scatter value of the sample. For determination of the scatter value, a histogram is constructed, which displays the frequency of a certain scatter value versus the corresponding scatter data. In this diagram, the Total Scatter value is defined as the value of highest probability. Also, the lower and the upper scatter value with a relative probability value of $90 \%$ next to frequency maximum are considered to supply additional information on the width of the distribution. The measurements shall be performed in a clean room environment and are 
calibrated with a diffuse reflectance standard of known Lambertian scatter characteristic. Before publication, the practicability of ISO 13696 had been tested in different national and international round-robin tests with success. For example, the comparability of the two measurement concepts had been verified within a serial national measurement campaign at the wavelength $633 \mathrm{~nm}$ [30] on special model samples. In addition, an extended international parallel measurement campaign involving ten partners and identical sample sets of practical laser components (high reflecting mirrors, beam splitters and laser windows) had been organized [31].

For characterization of specimens with scatter values typically below $10 \mathrm{ppm}$, the zero signal of the system with unloaded sphere has to be determined and shall range below a fraction of $1 \mathrm{ppm}$. In this regime, Rayleigh scattering in the volume of the sphere may already contribute to the zero signal and must be suppressed by flushing with appropriate gases or reduction of the atmospheric pressure. When approaching the DUV-range, no stable material with sufficient diffuse reflectivity for the integration mechanism in Ulbricht-spheres is available. Below 200nm and even in the EUV spectral range, Coblentz-hemispheres coated with adapted high reflecting metal and protection layers are preferred for TS-measurements. In comparison to the well-defined integration principle in the Ulbricht-sphere, the collection mechanism of the Coblentz-sphere can be described by a conjugate imaging of the test surface onto the detector, where the Coblentz-hemisphere acts as a spherical mirror. Since the imaging involves also acceptance angles near to $90^{\circ}$, severe aberrations have to be taken into account for an estimation of the measurement accuracy [32]. Before the background of present trends in photonics to radiation sources and applications with ever decreasing wavelengths down to the BEUV spectral range, problems related to the poor imaging quality and the calibration of Coblentz-spheres are gaining of increasing importance. In this context, ISO 13696 is presently under observation and discussed in view of a revision reflecting the technical progresses of the last 15 years.

In view of the essential role of optical scattering in modern photonic devices, additional standardization activities had been initiated during the last five years. The project ISO/CD 19962, "Spectroscopic measurement methods for integrated scattering by plane parallel optical elements", which is presently in the state of a Committee Draft (CD), is dedicated to spectrophotometric measurements of moderate scatter values. The fundamental measurement arrangement consists of a spectrophotometer with an Ulbricht-sphere which is integrated in the system. For a measurement of the forward scattering of a specimen, four cycles are prescribed. In the first step, the baseline of the system is recorded as a function of the wavelength with a $100 \%$ diffuse reflectance standard attached to the exit port of the Ulbricht-sphere. Then the sample is mounted at the entrance port of the sphere, and the spectral transmittance $T_{\text {st }}(\lambda)$ including scattering is measured. The subsequent cycle is performed with open exit port of the sphere to determine the spectral scattering $T_{\text {ss }}(\lambda)$. Finally, the spectral signal $\mathrm{T}_{\mathrm{rs}}(\lambda)$ of the empty sphere is monitored for an estimation of the measurement uncertainty. The spectral forward scattering $\mathrm{S}_{\text {for, MCA }}\left(\lambda_{\mathrm{i}}\right)$ is then calculated by the following equation for each wavelength value $\lambda_{\mathrm{i}}$ :

$$
s_{f o r, M C A}\left(\lambda_{i}\right)=\frac{T_{s s}\left(\lambda_{i}\right)-T_{s t}\left(\lambda_{i}\right) T_{r s}\left(\lambda_{i}\right)}{1-T_{r s}\left(\lambda_{i}\right)}
$$

The concept of ISO/WD 19962 is devoted to measurement with medium accuracy predominantly for the visible spectral range between 350 and $850 \mathrm{~nm}$ according to the parameters of the spectrophotometer and the detector unit. Typical error margins include a wavelength accuracy of $\pm 0.2 \mathrm{~nm}$, photometric fluctuations below $\pm 0.3 \%$, and nonlinearities of $0.6 \%$, respectively.

A second standardization project, ISO/WD 19986, "Test method for angle resolved scattering", is concentrated on the measurement of angle resolved scattering (ARS) on the basis of a goniometric system and detectors moved around the irradiated sample. The principles described in this Working Draft (WD) are generally similar to the former ARS standards with some practical modifications to improve the versatility and practicability of the measurement routine and evaluation techniques.

\subsection{Measurement of Laser Induced Damage Thresholds (LIDT)}

The power handling capability of optical components and materials is still continuing to be of outmost importance for the development of laser systems and their applications since the invention of the first lasers at the beginning of the 1960es. Following the history of laser development, a text passage from an interesting interview of Dr. Fred McClung can be cited [33]: "Ted made his first laser by vacuum depositing a silver coating on the ends of the crystal and then scratching a hole of about a millimeter diameter in the soft silver coating". In this context one of the first observations of laser damage may be also attributed to the scientific work of McClung [34] in 1961, where he reported laser damage effects 
observed in the silver coatings of his ruby laser. Another early mention of laser damage to optical coatings from the viewpoint of applications in material processing can be found in a publication of A. Schawlow [35] in 1963 with the citation: "Moreover, a laser of even medium power is energetic enough to melt a small amount of any substance. This can be useful for welding and vaporization, but it places severe constraint on the construction of the mirrors and the lasers. Sometimes internal reflection in prisms is used, to avoid the need for thin reflecting films which are easily damaged." The problem of power handling capability persisted of the following years, and in 1969 the first Boulder Damage Symposium [36] was held, which now celebrated its $50^{\text {th }}$ birthday in September, 2018.

During the early days of the Symposium, the comparability of laser damage threshold data was always discussed and revealed significant discrepancies among the research laboratories. Before this background, an international round-robin test on damage thresholds of dielectric mirrors for the Nd:YAG-lasers was conducted in 1981 [37], which indicated enormous deviations by orders of magnitude among the involved research groups. This was the motivation for the beginning of an activity for a standardization of LIDT-measurements on the ISO-level. After a series of extended discussion, a first DIS, that described a 1 on1 damage test, was launched for voting in ISO/TC 172/ SC 9 "Optics and Photonics Electro-optical Systems", at the end of 1991. However during the balloting period, further decisions were made to completely remodel this first DIS-version and to include also an S on 1 test that is of more significance for optical laser components in practice. Considerable efforts concerning the definition of damage, details of the measurement procedure and protocol, practical testing as well as extended round robin tests $[38,39]$ were necessary, until the first two parts of the ISO standard, ISO 11254, could be published in the years 2000 and 2001 [6]. However, during the elaboration of these standards the need for a third part dedicated to an assurance of laser components in respect to a certain laser power handling capability was perceived, and a restructuring of the first two parts in respect to the actual developments of laser technology was considered. Consequently, the third part of ISO 11254-3, "Assurance of laser power (energy) handling capabilities" became available in 2006 [6], and the discussion on the format of the standard also involving further roundrobin campaigns [40] continued during the last decade. As an outcome a new renumbered standard series on LIDT measurements, ISO 21254 [7], was published in 2011:

ISO 21254 1-3 and ISO/TR 21254-4, Test methods for laser induced damage threshold of optical surfaces -

Part 1: Definitions and general principle 2011/07

Part 2: Threshold determination 2011/07

Part 3: Assurance of laser power (energy) handling capabilities 2011/07

Part 4: Technical report: Inspection, detection and measurement, 2011/9

This remodeled standard series features measurement techniques similar to the concepts described in the former version, however it had been restructured and extended by several technical details as well as an additional technical report dedicated to practical aspects of damage inspection and detection techniques. During the last 15 years the standard gained many applications in the community and is now established as a mandatory catalogue entry in the quality management systems of laser components manufacturers as well as in research.

The fundamental principles for the determination of LIDT values impose severe restrictions on the beam parameters of the test facility and the evaluation algorithms to achieve comparable measurement results. Whenever possible, laser systems operating in single transversal and longitudinal modes with well-defined beam parameters are recommended. For a reliable characterization of the laser beam, an adapted beam diagnostic package has to be installed that monitors the beam parameters directly at the specimen or a conjugate location within the damage test facility. Besides on-line damage detection systems, an inspection procedure using a differential interference contrast microscope is prescribed for a reliable assessment of the damage state of the irradiated sites on the test surface. In the 1 on 1 measurement protocol, each site on sample surface is irradiated by a single pulse of defined energy, which is varied to cover values with confident survival up to always positive damage of the surface during the test. After the mandatory ex-situ inspection of the specimen, a data set consisting of energy values and the assigned damage state is obtained. Before the background of fundamental studies on the statistical behavior of damage [41, 42], the evaluation of the raw data is based on an extrapolation of the so-called survival curve. For construction of the survival curve for a damage measurement protocol, the damage probability is plotted versus the power or energy density of the test pulses. According to ISO 21254, the LIDTvalue is determined by extrapolating the survival curve to highest fluence with a damage probability of zero. Even though 1 on 1-LIDT-data are not representative for typical applications of optical components, they are listed in many catalogues of optics manufacturers and are often discussed within fundamental investigations in the damage mechanisms of optical components. 
The more practical $\mathrm{S}$ on 1 threshold is determined on the basis of irradiation sequences with repetitive laser pulses of identical properties. The standard prescribes an interrogation of each site on the test surface with such a train of pulses, where the energy of the so-called typical pulse representing the average pulse parameters is tuned in a range adequate for the damage behavior of the sample. Since damage may occur already after very few pulses, an online damage detection system has to be installed, that interrupts the laser system to avoid catastrophic damage of the specimen and enables the determination of the number of pulses until damage occurs. The evaluation of the measurement data involves the deduction of survival curves for selected pulse numbers and results in characteristic damage curves that display the energy density values of selected damage probabilities values $(0 \%, 10 \%, 50 \%)$ plotted as a function of the number of pulses. This diagram gives an overview on the damage behavior of the specimen and allows also for a rough estimation of its lifetime via on an algorithm described in the standard. The third part of the standard had been elaborated during comprehensive studies on the statistical nature of damage effects [43] and describes a nondestructive certification of an optical component in respect to a defined power or energy density level. The fundamental approach consists of an irradiation of a defined fraction of the test surface with pulses of preselected energy in a raster scan. In case no damage is detected, the sample is certified for the selected energy with a risk level that is directly dependent on the fraction of the irradiated surface and the number of allowable vulnerable defects. As an example, a risk of $10 \%$ for the case that the sample carries one defect, which is damaged at a lower energy level than certified, is according to a surface fraction of approximately $90 \%$ of the entire inspection area. In this context, the overlap factor is an additional test parameter that has to be considered especially for Gaussian beams and may influence the test result due to preconditioning effects. For more detailed descriptions of the measurement routines further material is available in [44].

In the time period after publication of the latest version of ISO 21254, several new aspects of damage testing were discussed in the community especially focusing on the ns-regime where laser damage is often driven by defects or inclusions with a statistical distribution of size, material, and location in the test component. Since the typical S on 1 test only covers a relatively small part of the test surface, the uncertainty is occasionally too high to provide useful information to the manufacturer or user of a laser component. Besides other recent achievements in operation parameters and conditions, this deficiency motivated a further revision of the LIDT standard which was started as a new work item in ISO/TC 172/SC 9/WG 1 "Terminology and test methods for electro-optical systems" in the year 2015. In order to accomplish a sustainable revision, the community was asked to file in comments to the working group at different occasions as for example also within a round table discussion organized at the Laser Damage Symposium 2016 [45]. All comments compiled until then were thoroughly discussed and evaluated during the last meeting of WG 1 at the end of the year 2017.

a)

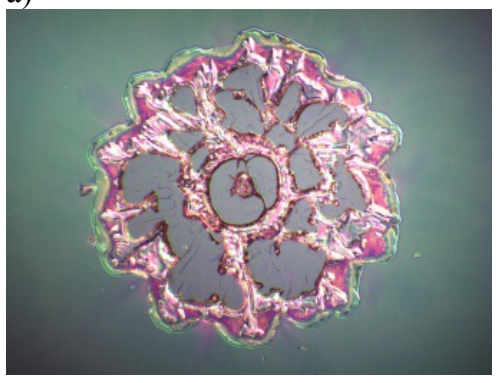

b)

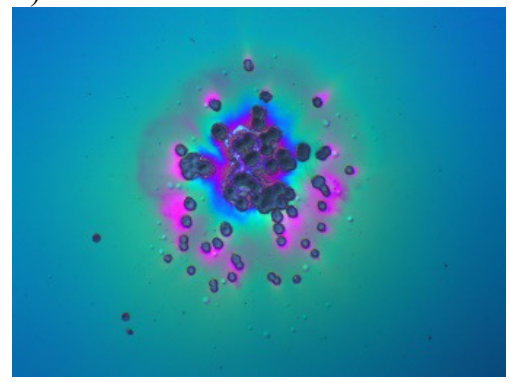

c)

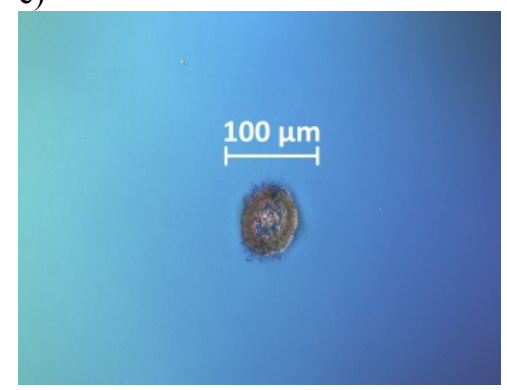

Fig. 3: Examples for damage morphologies in the long pulse (a, dominated by thermal effects and coating absorption), short pulse (b, damage initiated by defects and inclusions), and ultra-short pulse (c, governed by electronic mechanisms) duration regime. 
As major outcome a list of items for revision was created which will be considered for the future work of the standardization group. A few selected points of outmost importance are summarized in the following:

- The R-on-1 test procedure [46] shall be integrated in the ISO Standard, because it offers several advantages for testing of components with limited space. In contrast, preconditioning effects [47] have to be taken into account that may result in an overestimation of the power handling capability for specific components.

- Adapted pre-testing schemes are recommended to identify the underlying damage mechanism in a sample for a more efficient subsequent testing routine (see for example Fig. 3 and Fig.4).

- Tests with random fluence variation shall be considered in conjunction with alternative data reduction techniques including the Bayesian approach [48] and the cumulative method [49] to improve the accuracy and efficiency of test.

- The test parameters and operation conditions mentioned in the standard shall be updated in respect to the properties of recently developed laser sources: beam shape, especially cw-operation schemes, and different sample geometries.

- Further ideas are concentrated on the conception of a special dummy data package containing typical damage test raw data that can be employed at the testing laboratories for an analysis of their specific data reduction techniques.

Especially for the aspect of defect induced damage in the ns-regime a new approach is conceived by the US-National Committee TF 7 within the Optics and Electro-Optics Standards Council and will be integrated in the part 3 of the revised ISO 21254-series [50]. Furthermore, an additional measurement technique is well established in some research laboratories, the raster scan [51], and shall be integrated in the upcoming revised ISO-standard.

a)

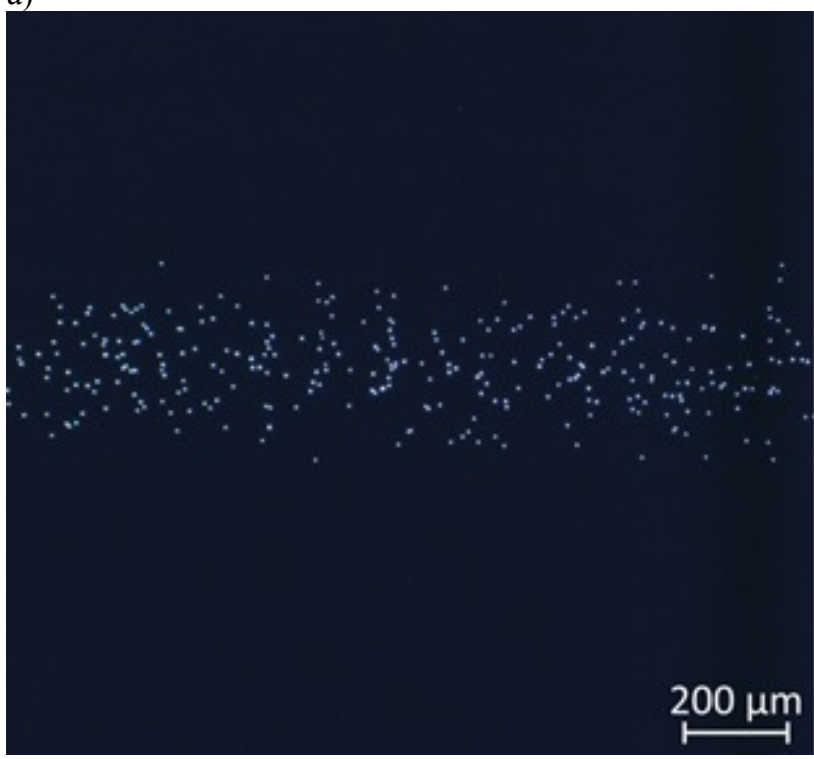

b)

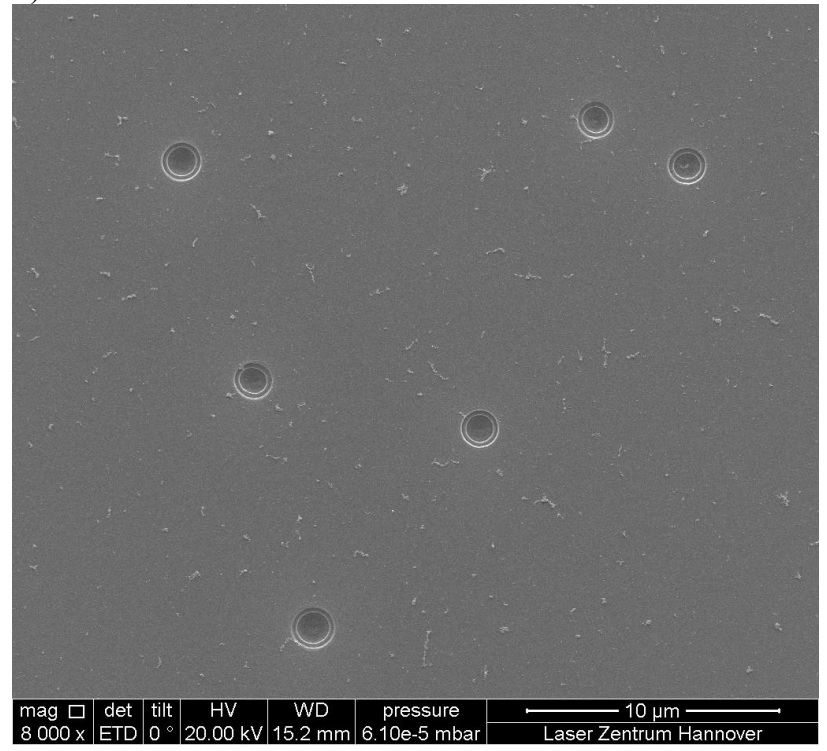

Fig.4: Flat bottom pit damage morphology that is widely independent of irradiation fluence and pulse number [61]. Dark field microscopy image of a line scan on an antireflective coating for $355 \mathrm{~nm}$ (a), and SEM image of typical damage sites of the AR-coating (b). Laser parameters: $355 \mathrm{~nm}, 5.3 \mathrm{~ns}, 19.4 \mathrm{~J} / \mathrm{cm}^{2}$ (a) and $14.2 \mathrm{~J} / \mathrm{cm}^{2}$ (b). 
However this concept has to be evaluated in respect to a variety issues mentioned in comments of the community concerning the revision of the standard (selection of original comments to WG 1):

- Definition of damage (crater or pinpoint) is not clear as well as detection algorithm or particle calculation method as well as resolution (the pertinent publication is not dealing with that).

- Definition of „damage density“ is not accurate, because in the present form it is a damage density per effective area (without considering overlap).

- Overlap parameter is an open question, because in the points between sample sees exactly two pulses.

- Dealing with ablation particles and dust is not considered (we don't know yet how, but some recommendations perhaps are necessary).

- Need for round-robin tests based on such raster scan procedure.

In modern laser systems, optical surfaces may be operated at different wavelengths simultaneously. Recent studies [52, 53] imply a significant reduction of the damage thresholds at the individual wavelengths for such operation conditions. Consequently, the power handling capability of a specific component will be overestimated, if the threshold values for single wavelength operation are presumed for the design of laser systems. A study [54] dedicated to fs-pulses at the wavelength $780 \mathrm{~nm}$ and its second harmonic reveals a reduction by a factor of two for the threshold of the fundamental if the second harmonic is simultaneously applied with fluence near to the damage threshold. The corresponding problems for a standardization of LIDT values under operation conditions involving more than one wavelength have to be discussed for a revision of ISO 21254. Also it needs to be pointed out that the topic of laser damage in optical fibers gains of importance in many applications. Especially in laser surgery, where fibers are often employed for delivery of the laser beam directly to the patient, laser damage may lead to fatal hazards and shall be avoided under all circumstances. In view of the enormous cost pressure imposed on hospitals nowadays and the pricing of high quality fibers, a qualification standard for medical fibers is urgently needed. Presently, a corresponding standardization project is elaborated in ISO/TC 172/SC 9/WG 1 "Laser systems for medical applications". Especially for applications in surgery, the environmental conditions often feature a high percentage of oxygen, which may support the initiation of laser damage and maintain burning of the fiber afterwards. These special circumstances have to be considered for the new standardization project ISO/AWI 22248 "Laser and laser-related equipment - Test methods for laser-induced damage threshold Classification of medical laser delivery and application systems" [55]. However, also in laser material processing and other application areas with higher power levels but lower environmental demands, research work [56, 57] on the power handling capability of fibers and the development of an adapted standardized measurement procedure moves into the focus. Research activities [58] are presently also extended to testing procedures of polymer fibers [59] and will propel the development of a new standardization project on fiber damage (see Fig. 5).

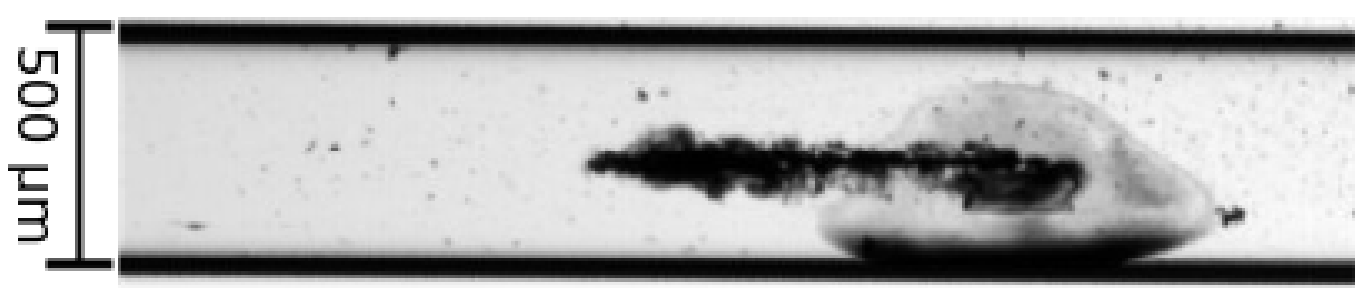

Fig. 5: Damage morphology observed in a PMMA fiber under exposure to laser radiation at $532 \mathrm{~nm}$ and a pulse duration of 7.3ns [59].

\section{CONCLUSIONS AND OUTLOOK}

After more than two decades, international standardization in optics characterization has achieved major progresses and generated a critical set of standards established in industry, research and commerce. For most relevant quality parameters of laser components and laser beams, measurement concepts are available and applied on a routine basis. Besides the observation of new trends in laser technology and the initiation of according standardization projects, the maintenance and technical revision of the existing standards is one of the essential tasks of the involved standardization committees. Presently, major activities are concentrated on a complete revision of the LIDT standard ISO 21254, an adaptation of the 
laser calorimetry standard ISO 11551 in respect to different sample geometries, and an extension of the Total Scatter measurement standard ISO 13696 to shorter wavelengths. These demanding tasks will require enormous efforts especially focused on a detailed understanding and thorough testing of the measurement methods within extended roundrobin tests. Furthermore, the standard series ISO 9211 [10], which is dedicated to the properties and test methods of optical coatings, is subject of a comprehensive modernization and will be widened in its scope to laser applications with four additional parts. Concrete work items accepted by the ISO committee members are concentrated on the two described standards for the spectrophotometric measurement of optical scattering, ISO/CD 19962, and angle resolved scattering, ISO/WD 19986, as well as an extension of the cavity ring down standard ISO 13142 with transmittance measurements. Furthermore, a vivid discussion on the implementation of photothermal techniques for mapping of absorption in laser components started in the last year. Standardization project discussed in view of future trends and needs in laser technology include the topics of cleaning, storage, and transportation as well as lifetime and degradation effects of laser components.

\section{ACKNOWLEDGMENTS}

The authors thank the European Regional Development Fund for the financial support of the joint project LAPOF (contract no. ZW 685003502) and the Sino-German Center for Research Promotion (contract GZ 1275), the Deutsche Forschungsgemeinschaft (DFG, German Research Foundation, contract RI 645/8-1), and the National Natural Science Foundation of China (NSFC).

\section{REFERENCES}

[1] ISO 15368: Optics and optical instruments -- Lasers and laser related equipment -- Measurement of reflectance of plane surfaces and transmittance of plane parallel elements. International Organisation for Standardisation, Geneva, 2001

[2] ISO 24013: Optics and photonics -- Lasers and laser-related equipment -- Measurement of phase retardation of optical components for polarized laser radiation. . International Organisation for Standardisation, Geneva, 2006

[3] ISO 11551: Optics and optical instruments -- Lasers and laser related equipment -- Test method for absorptance of optical laser components. International Standard. International Organisation for Standardisation, first version 1997, revised version published 2003, Geneva, 2003

[4] ISO 13696: Optics and optical instruments -- Lasers and laser related equipment -- Test Method for Radiation Scattered by Optical Components. International Organisation for Standardisation, Geneva, 2002

[5] ISO 13142: Electro-optical systems -- Cavity ring-down technique for high-reflectance measurement, International Organisation for Standardisation, Geneva, 2015

[6] ISO 11254: Optics and optical instruments -- Lasers and laser related equipment -- Test methods for laser induced damage threshold of optical surfaces. Part 1: 1 on 1-test, 2000, Part 2: S on 1 test, 2001, Part 3: Assurance of laser power handling capabilities, 2006, International Organization of Standardisation, Geneva

[7] ISO 21254: Optics and optical instruments -- Lasers and laser related equipment -- Test methods for laserinduced damage threshold, Part 1: Definitions and general principle, Part 2: Threshold determination 2011/07, Part 3: Assurance of laser power (energy) handling capabilities 2011/07, Part 4: Inspection, detection and measurement, 2011/9, International Organization for Standardization, Geneva, 2011

[8] ISO 10110: Preparation of drawings for optical elements and systems. Part 1: General, Part 2: Material imperfections - Stress birefringence, Part 3: Material imperfections - Bubbles and inclusions, Part 4: Material imperfections - Inhomogeneity and striae, Part 5: Surface form tolerances, Part 6: Centering tolerances, Part 7: Surface imperfection tolerances, Part 8: Surface texture; roughness and waviness, Part 9: Surface treatment and coating, Part 10: Table representing data of optical elements and cemented assemblies, Part 11: Non-toleranced data, Part 12: Aspheric surfaces, Part 14: Wavefront deformation tolerance, Part 17: Laser irradiation damage threshold, Part 19: General description of surfaces and components. International Organization for Standardization, Geneva 1996-2018

[9] ISO 9022: Optics and optical Instruments -- Environmental test methods. Part 1: Definitions, extent of testing, Part 2: Cold, heat and humidity, Part 3: Mechanical stress, Part 4: Salt mist, Part 6: Dust, Part 7: Resistance to drip or rain, Part 8: High internal pressure, low internal pressure, immersion, Part 9: Solar radiation and 
weathering, Part 11: Mould growth, Part 12: Contamination, Part 14: Dew, hoarfrost, ice, Part 17: Combined contamination, solar radiation, Part 20: Humid atmosphere containing sulfur dioxide or hydrogen sulfide, Part 22: Combined cold, dry heat or temperature change with bump or random vibration, Part 23: Low pressure combined with cold, ambient temperature and dry or damp heat. International Organization for Standardization, Geneva 1996-2018

[10] ISO 9211: Optical Coatings, Part 1: Definitions, Part 2: Optical properties, Part 3: Environmental durability, Part 4: Specific test methods, Part 5: Minimum requirements for antireflecting coatings, Part 6: Minimum requirements for reflecting coatings, Part 7: Minimum requirements for neutral beam splitting coatings, Part 8: Minimum requirements for coatings used for laser optics (parts in different draft stages or published). International Organization for Standardization, Geneva

[11]ISO/TR 20811: Optics and photonics -- Lasers and laser-related equipment -- Laser-induced molecular contamination testing. International Organization for Standardization, Geneva 2017

[12] A. C. Boccara, D. Fournier, and J. Badoz, Appl. Phys. Lett. 36, 130, 1980, and A. C. Boccara, D. Fournier, W. Jackson, and N. M. Amer, Opt. Lett. 5, 377, 1980

[13] M. Gibbs and R. Wood: Laser induced damage of mirror and window materials at 10.6 $\mu \mathrm{m}$. Laser Induced Damage in Optical Material, NBS SP 462, pp.181-188, 1976

[14]D. Ristau, U. Willamowski, and H. Welling: Measurement of optical absorptance according to ISO 11551: Parallel round-robin test at $10.6 \mu \mathrm{m}$. SPIE Proceedings of the 30th Symposium on Laser Induced Damage in Optical Materials, SPIE Vol.3578, pp. 1998

[15]B. Steiger, U. Pfeifer, P. Meja, U, Broulik, V. Neumann: Practicability of absorption measurements according to ISO/DIS 11551, Proceedings of the International Workshop of Laser Beam and Optics Characterization. SPIEVol. 2870, pp.495-501, 1996

[16] U. Willamowski, T. Groß, D. Ristau and H. Welling: Calorimetric measurement of optical absorption and transmissivity with sub ppm sensitivity, In Proc. of SPIE, vol. 2775 (edited by A. E. Gee and J.-F. Houee), pp. 148-158, Specification, Production, and Testing of Optical Components and Systems, 1996

[17] U. Willamowski, D. Ristau and E. Welsch: Measuring the absolute absorptance of optical laser components, Appl. Opt., 37 (36): pp. 8362-8370, 1998

[18]E. Eva and K.R. Mann. Nonlinear absorption phenomena in optical materials for the UV-spectral range, Proceedings of the International Workshop of Laser Beam and Optics Characterization. SPIE Vol. 2870, pp.476-482, 1996

[19] O. Apel, K. Mann, J. Heber, and R. Thielsch: Nonlinear absorption phenomena in oxide coatings for $193 \mathrm{~nm}$. Proceedings of the 31th Symposium on Laser Induced Damage in Optical Materials, SPIE Vol. 3902, pp.235241, 242-249, 2000

[20] H. Blaschke, D. Ristau, E. Welsch and O. Apel: Absolute Measurements of non-linear Absorption near LIDT at 193 nm, Proc. of SPIE (edited by G. J. Exarhos, A. H. Guenther, M. R. Kozlowski, K. L. Lewis, and M. J. Soileau), Proceedings of the 32th Annual Symposium of Laser Induced Damage in Optical Materials, SPIE Vol. 4347, pp. 447-453, 2001

[21]H. Blaschke, S. Günster and D. Ristau: The influence of different cleaning procedures on the spectral performance of optical components in the DUV spectral range, Proc., WLT, 6th International Workshop of Laser Beam and Optics Characterization, 2001

[22] I. Balasa, H. Blaschke, L. Jensen and D. Ristau: Impact of $\mathrm{SiO} 2$ and $\mathrm{CaF} 2$ surface composition on the absolute absorption at 193nm, Proc. SPIE (edited by G. Exarhos, V. Gruzdev, J. Menapace, D. Ristau, and M. Soileau), , Proceedings of the 43nd Annual Symposium on Optical Materials for High Power Lasers, SPIE Vol. 8190, p. 81901T, 2011

[23] I. Balasa, L.O. Jensen and D. Ristau: Laser calorimetric absorptance testing of samples with varying geometry, Optical Engineering, 53(12):122503, 072014

[24] G.M. Bennett and L. Mattsson: Introduction to Surface Roughness and Scattering. Second Edition (Optical Society of America, Washington D.C. 1999), ISBN 1-55752-609-5

[25] A. Duparré: Light Scattering of Thin Dielectric Films. In Handbook of Optical Properties, Volume 1: Thin Films for Optical Coatings. Eds.: R.E. Hummel and K.H. Guenther, CRC Press, 1995 
[26] ASTM Doc. F1048-87: Standard test method for measuring the effective surface roughness of optical components by total integrated scattering, August 1987 recent version: ASTM F 1048-87 (1999)

[27] ASTM Doc. E1392-90: Standard practice for angle resolved optical scatter measurements on specular or diffuse surfaces. November 1990, recent versions: ASTM E 1392-96 (2002), withdrawn 2003, now SEMI-Guideline: SEMI ME1392-1109: Guide for Angle Resolved Optical Scatter Measurements on Specular or Diffuse Surfaces

[28] R. Ulbricht: Die Bestimmung der mittleren räumlichen Lichtintensität durch nur eine Messung. Elektrotech. Zeit 29, 595, 1900

[29] W.W. Coblentz: Natl. Bur. Stand. (U.S.) Bull.9, p.283, 1913

[30] M. Kadkhoda, P. Strink, and D.Ristau, A. Duparré and S. Gliech, N. Reng and M.Greif, R. Schuhmann and M. Goldner: Concepts for Standardisation of total scatter measurements at 633. Proceedings of the 4th International Workshop on Laser, Beams and Optics Characterisation, p. 298-313, VDI-Verlag, 1998

[31] P. Kadkhoda, A. Müller, D. Ristau, A. Duparré, S. Gliech, H. Lauth, U. N. Reng, M. R. Schuhmann, C. Amra, C. Deumie, C. Jolie, H. Kessler, T. Lindström, C. G. Ribbing and J. M. Bennett: International round-robin experiment to test the ISO total scattering draft standard, Appl. Opt., 39 (19): pp. 3321-3332, 2000

[32] P. Kadkhoda, A. Müller, D. Ristau: Total scatter losses of optical components in the DUV/VUV spectral range. Proceedings of the 31th Symposium on Laser Induced Damage in Optical Materials, SPIE Vol.3902, pp.118127, 1999

[33] https://www.aip.org/history-programs/niels-bohr-library/oral-histories/5041, vis. 10/2018

[34]F.J. McClung and R.W. Hellwarth: Giant optical pulsation from Ruby, J. Appl. Phys. Vol.33, No.3, 1962

[35] A.L. Schawlow: Optical and Infra-red Masers, Contemporary Physics, 5:2, 81-102, 1963

[36] Proceedings of the Conference Damage in Laser Glass, ASTM STM STP 469 (1969)

[37] K.H. Guenther, J. Ebert, E. Kiesel et.al.: 1.06- $\mu$ m laser damage of thin film optical coatings: a round-robin experiment involving various pulse lengths and beam diameters. Applied Optics Vol.23, p.3743, 1984

[38] W. Riede, U. Willamowski, M. Dieckmann, D. Ristau, U. Broulik, B. Steiger: Laser induced damage measurements according to ISO/DIS 11254-1: Results of a national round robin-test on Nd:Yag laser optics SPIE Proceedings of the 29th Symposium on Laser Induced Damage in Optical Materials, Vol.3244, pp.96-105, 1997

[39] J. W. Arenberg, W. Riede, U. Broulik, B. Steiger, U. Willamowski, and D. Ristau: National round-robin test on laser induced damage at $1.064 \mu \mathrm{m}$ : Revised data reduction and correlation analysis. SPIE Proceedings of the 30th Symposium on Laser Induced Damage in Optical Materials, SPIE Vol.3578, pp.645-656,1999

[40] K. Starke, D. Ristau, S. Martin, A. Hertwig, J. Krüger, P. Allenspacher, W. Riede, S. Meister, C. Theiss, A. Sabbah, W. Rudolph, V. Raab, F. Grigonis, T. Rakickas and V. Sirutkaitis: Results of a Round-Robin Experiment in Multiple-Pulse LIDT Measurement with Ultrashort Pulses, Proceedings of the 35th Annual Symposium on Optical Materials for High Power Lasers (edited by G. J. Exarhos, A. H. Guenther, N. Kaiser, K. L. Lewis, M. J. Soileau, and C. J. Stolz), SPIE Vol.5273, pp.281-287, 2004

[41] S.C. Seitel and J.O. Porteus: Towards improved accuracy in limited scale laser damage testing via the "onset method”. NBS Spec. Publ.6988, p.502, 1983

[42] M.F. Becker, C. Ma, and R.M. Walser: Multiple pulse laser induced failure prediction for Mo metal mirrors. SPIE Proceedings of the 22nd Symposium on Laser Induced Damage in Optical Materials, SPIE Vol.1441, pp.174-187, 1990

[43] J.W. Arenberg: Spatial fluence profile for certification test of laser damage resistance: Call for comments. SPIE Proceedings of the 28th Symposium on Laser Induced Damage in Optical Materials, SPIE Vol.2966, pp.531534, 1996

[44]D. Ristau: Laser Damage in Optical Materials, CRC Press, ISBN 9781439872161, 2014

[45] D. Ristau: Standardization Round-Table Discussion, in collocation with the $48^{\text {th }}$ Laser Damage Conference, September $26^{\text {th }}, 2016$

[46] J.P. Hue Garrec, J. Dijon, and P. Lyan: R-on-1 automatic mapping: A new tool for laser damage testing. Proc. SPIE Vol.2714, pp.90-101, 1996

[47] C.R. Wolfe, M.R. Kozlowski, J.H. Campbell, F. Rainer, A.J. Morgan, and R.P. Gonzales: Laser Conditioning of Optical Thin Films. Proc. SPIE Vol.1438, pp.360-375, 1989 
[48] G. Bataviciute, P. Grigas, L. Smalakys, and A. Melninkaitis, Bayesian approach of laser-induced damage threshold analysis and determination of error bars, Boulder Damage Symposium 23-26 September 2012, Boulder, Colorado, USA, SPIE Vol.8530, pp.85301S, 2012

[49] L. O. Jensen, M. Mrohs, M. Gyamfi, H. Mädebach, D. Ristau, Higher certainty of the laser-induced damage threshold test with a redistributing data treatment. Review of Scientific Instruments 10 (86), 103106, 2015

[50] J.W. Arenberg, D. Howland, M.D. Thomas, T. Turner, and A. Krisiloff: U.S. National Committee proposed revision to the ISO Laser Damage Standard: 2018 Progress Report, publication 10805-06 in these proceedings, 2018

[51] R. Chow, M. Runkel, and J.R. Taylor: Laser damage testing of small optics for the National Ignition Facility. Appl. Opt. Vol.44, pp.3527-3531, 2005

[52] M. Gyamfi, P. Jürgens, M. Mende, L.O. Jensen and D. Ristau: Dual-wavelength ultra-short pulse laser damage testing, SPIE Laser Damage, SPIE Vol.9237, pp.92371Q, 2014

[53] M. Mrohs, L. O. Jensen, S. Günster, T. Alig, D. Ristau, Dual wavelength laser-induced damage threshold measurements of alumina/silica and hafnia/silica ultraviolet antireflective coatings. Applied Optics Vol.55, pp.104-109, 2016

[54] M. Gyamfi, P. Jürgens, L. O. Jensen and D. Ristau. Delay dependency of two-pulse femtosecondlaser damage. SPIE 9632, Laser-Induced Damage in Optical Materials, SPIE Vol.9632, pp.96320W, 2015

[55]H.-P. Berlien: Test Methods for Laser-induced Damage Threshold of Medical Laser Delivery and Applications Systems (Plenary Presentation), publication 10805-4, in these proceedings, 2018

[56] G. Mann, J. Vogel, R. Preuß, P. Vaziri, M. Zoheidi, M. Eberstein, and J. Krüger: Nanosecond laser damage resistance of differently prepared semi-finished parts of optical multimode fibers, Applied Surface Science Vol.254(4), pp.1096-1100, 2007

[57] G. Mann, J. Vogel, R. Preuß, P. Vaziri, M. Zoheidi, M. Eberstein, and J. Krüger: Nanosecond laser-induced surface damage of optical multimode fibers and their preforms, Applied Physics A Vol.92, pp.853-857, 2008

[58] G. Mann, M. Zoheidi, and J. Krüger: High performance fibers at the limit, Laser-induced damage reveals the performance limit of optical fibers, Laser+Photonics Vol.4, pp.18-21, 2012

[59] K. Kiedrowski, I. Balasa, D. Kracht, D. Ristau: Determination of the laser-induced damage threshold of polymer optical fibers, publication 10805-83, in these proceedings, 2018

[60]Y. Willer, L. Hao, I. Balasa and D. Ristau: Calibration accuracy of laser calorimetry for common crystal geometries, Proc. of Laser-Induced Damage in Optical Materials 2017, SPIE Vol.10447, pp.104471V, 2017

[61] S. Paschel, I. Balasa, L. Jensen, D. Ristau, X. Cheng, Z. Wang: Nanopits and catastrophic damage, publication $10805-57$, in these proceedings, 2018 